\title{
Surface Stress Anisotropy of Ge(001)
}

\author{
M. T. Middel, H. J. W. Zandvliet,* and Bene Poelsema \\ Faculty of Applied Physics, MESA+ Research Institute, University of Twente, P.O. Box 217, \\ 7500 AE Enschede, The Netherlands
}

(Received 16 November 2001; published 29 April 2002)

\begin{abstract}
By analyzing the equilibrium shape of vacancy islands on the $\mathrm{Ge}(001)$ surface we have determined the surface stress anisotropy, i.e., the difference between the compressive stress component along the substrate dimer rows and the tensile stress component perpendicular to the substrate dimer rows. In order to extract the surface stress anisotropy we have used a model recently put forward by Li et al. [Phys. Rev. Lett. 85, $1922(2000)]$. The surface stress anisotropy of the clean $\mathrm{Ge}(001)$ surface is found to be $80 \pm 30 \mathrm{meV} / \AA^{2}$. This value is comparable to the surface stress anisotropy of the closely related $\operatorname{Si}(001)$ surface.
\end{abstract}

DOI: $10.1103 /$ PhysRevLett.88.196105

Among the most intriguing and frequently studied surfaces belong to the semiconductor group-IV (001) surfaces $[1,2]$. Because of its technological importance, the majority of studies have been devoted to the $\operatorname{Si}(001)$ surface rather than to the closely related $\mathrm{Ge}(001)$ surface. In many aspects the $\mathrm{Ge}(001)$ surface is very similar to the $\mathrm{Si}(001)$ surface. Both surfaces reconstruct to form rows of dimerized atoms, yielding a $(2 \times 1)$ surface unit mesh. The surface dimers can buckle with respect to the surface plane leading to higher-order reconstructions, such as $c(4 \times 2)$ and $p(2 \times 2)$. The dimerization of these surfaces in the $(2 \times 1), p(2 \times 2)$, or $c(4 \times 2)$ reconstruction induces an anisotropic surface stress tensor: the surface is under a compressive stress along the substrate dimer row direction and under a tensile stress in a direction perpendicular to the substrate dimer rows. If the surface exhibits a slight miscut with respect to the [001] direction, single layer steps are found. The monatomic steps separate terraces with alternating $(2 \times 1)$ and $(1 \times 2)$ reconstruction. After cleaning, $\mathrm{Ge}(001)$ exhibits a well-ordered domain pattern consisting of $(2 \times 1)$ and $c(4 \times 2)$ stripes and almost no missing dimer defects [3].

The missing dimer concentration on $\mathrm{Si}(001)$ can be increased by ion bombardment with noble gases [4-8], etching [9-13] $\left(\mathrm{O}_{2}, \mathrm{H}_{2}, \mathrm{Br}_{2}, \mathrm{I}_{2}\right.$, etc.), or metal contamination [14-18] $(\mathrm{Ni}, \mathrm{Cu}, \mathrm{Co}, \mathrm{Ag}$, etc.). For sufficiently high temperatures those missing dimers order into vacancy islands. The equilibrium shape at low vacancy concentrations $(<0.2-0.3$ monolayers) [19] of these vacancy islands is elongated in a direction perpendicular to the substrate dimer rows. At vacancy concentrations above $0.2-0.3$ monolayers the vacancy islands rotate shape and are aligned parallel to the substrate dimer rows [8]. The absence of dimerization at the bottom of the narrow vacancy islands in combination with the specific rebonding at the island edges is believed to be responsible for this shape transformation [19-24]. Ab initio calculations [20] and tight-binding total-energy calculations [21] are in perfect agreement with the experimental observations of the shape transformation. Such a shape transformation has not been observed for the $\mathrm{Ge}(001)$ surface. Experiments per-
PACS numbers: 68.35.Md, 68.37.Ef

formed on $\mathrm{Ge}(001)$ so far have revealed that the bottom of the vacancy islands is always dimerized and that the long axis of the vacancy islands is always aligned along the substrate dimer row direction [25]. The latter result is in agreement with the fact that the step edge formation energy of an $A$-type step edge is much lower than the step edge formation energy of a $B$-type step edge. However, as pointed out by Li et al. [26] the equilibrium shape of (vacancy) islands under stress [as in the case of $\mathrm{Ge}(001)$ and $\mathrm{Si}(001)]$ is determined not only be the step-free energies, but also by the strain energy. Thus, if one can measure the equilibrium shape of an (vacancy) island and if one knows the values of the step-free energies, one can, in principle, determine the strain energy (and hence the surface stress anisotropy). By analyzing the kink distributions we have recently extracted the step-free energies of both types of monatomic steps on Ge(001) [27]. Using these values and the equilibrium shape of a vacancy island we have for the first time determined the surface stress anisotropy of the dimer-reconstructed $\mathrm{Ge}(001)$ surface. Surprisingly, the surface stress anisotropy of $\mathrm{Ge}(001)$ is comparable to that of the closely related $\mathrm{Si}(001)$ surface $\left[80 \pm 30 \mathrm{meV} / \AA^{2}\right.$ for $\mathrm{Ge}(001)$ versus $70 \pm 10 \mathrm{meV} / \AA^{2}$ [28,29], $35 \mathrm{meV} / \AA^{2}$ [30], and $80-130 \mathrm{meV} / \AA^{2}$ [31] for $\mathrm{Si}(001)]$. It should be highlighted here that first principles calculations of the surface stress anisotropy of $\mathrm{Si}(001)$ result in very comparable surface stress anisotropies $[32,33]$.

Previously, Alerhand et al. [28] and Men et al. [29] have shown that application of uniaxial strain to the $\mathrm{Si}(001)$ surface leads to unequal $2 \times 1 / 1 \times 2$ domain population. From this relative domain population as a function of the applied strain these authors were able to extract the surface stress anisotropy. A second route to determine the surface stress anisotropy is to analyze the terrace width distribution [30,31]. Repulsive energetic and entropic step-step instructions will narrow the terrace width distribution. If one assumes that the surface stress anisotropy is the only cause for the energetic step-step interaction then the surface stress anisotropy can easily be determined from the terrace width distribution. However, both methods have their drawbacks: the first method is experimentally very 
complicated, whereas the second method relies on the assumption that the surface stress anisotropy is the only cause for the energetic step-step interaction. The model we put forward here is relatively simple and doesn't require any additional assumptions. Moreover, our method is also applicable to many other surface, including heteroepitaxial systems.

The experiments were performed with a scanning tunneling microscope (STM) in a vacuum system with a base pressure of about $1 \times 10^{-10}$ mbar. The Ge(001) samples were cleaned by cycles of $800 \mathrm{eV}$ argon-ion sputtering, annealing up to $1100 \mathrm{~K}$, and subsequent quenching to room temperature. This results in an atomically clean surface with less than $0.1 \%$ surface defects (mainly missing dimer defects). After the ion bombardment, cleaning, or annealing experiments the sample was transferred to the STM for

(a)
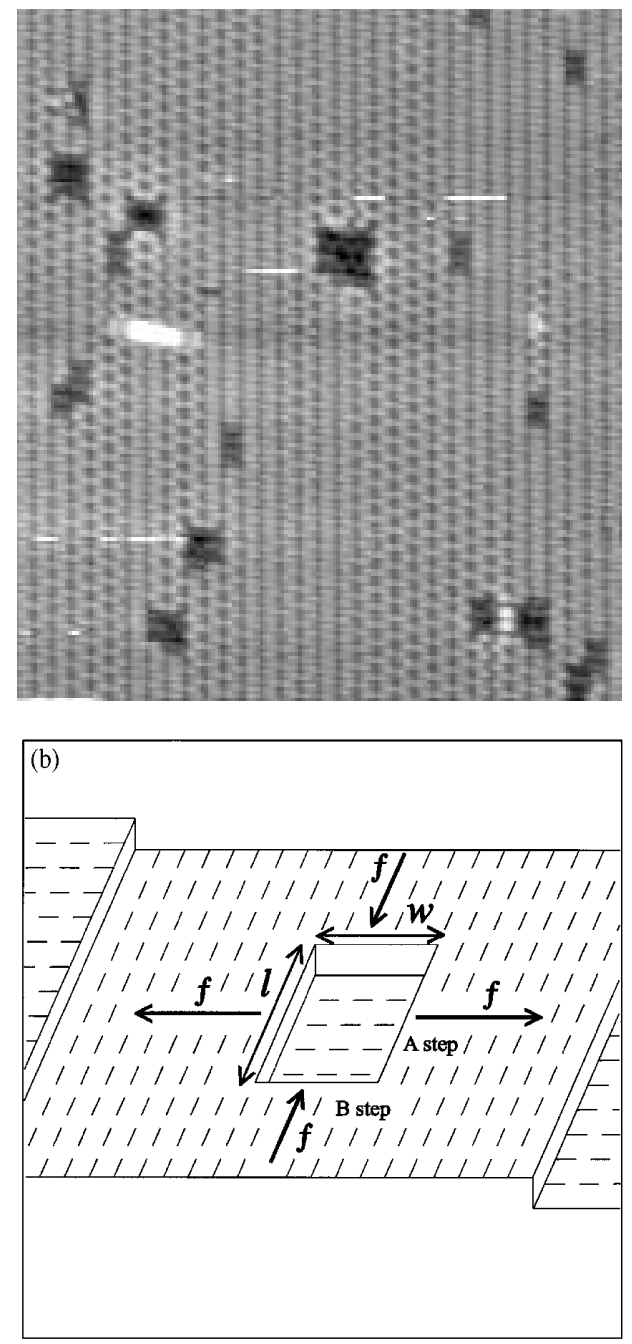

FIG. 1. (a) Typical filled-state STM image of a Ge(001) with a vacancy island. Sample bias - $1.6 \mathrm{~V}$, tunneling current $0.5 \mathrm{nA}$ and image size $20 \mathrm{~nm} \times 15 \mathrm{~nm}$. (b) Schematic drawing of a vacancy island, with a rectangular shape of length $l$ and width $w$. $f=\left(\sigma_{l}-\sigma_{w}\right)$ represents the elastic force monopole along the vacancy island periphery arising from surface stress anisotropy. imaging. The STM experiments were always performed at room temperature.

In order to obtain vacancy islands we first bombarded the $\mathrm{Ge}(001)$ surface at room temperature with $800 \mathrm{eV}$ argon ions with a total dose of about $(1-5) \times$ $10^{13}$ ions $/ \mathrm{cm}^{2}$ resulting in the removal of several percents of a monolayer of $\mathrm{Ge}$ followed by subsequent annealing at temperatures in the range from $400-700 \mathrm{~K}$. After room-temperature ion bombardment, the missing dimer defects, missing dimer defect clusters, and adatom clusters (mainly Ge dimers) are distributed randomly over the surface. Annealing for $1 \mathrm{~min}$ at a temperature of about $500 \mathrm{~K}$ is sufficient for the missing dimers and missing dimer defects to order themselves into larger vacancy islands ranging in sizes from 10 to 100 missing dimers.

With increasing annealing temperature the aspect ratio of the vacancy islands decreases slightly, which is in perfect agreement with the fact that the anisotropy of step-free energies decreases with increasing temperature. Therefore, we assume that the shape of the vacancy islands after annealing at a temperature above $500 \mathrm{~K}$ reflects the equilibrium shape reasonable well. In Fig. 1 an STM image of a $\mathrm{Ge}(001)$ surface bombarded with a low dose of argon ions and subsequently annealed at $500 \mathrm{~K}$ is depicted. The vacancy islands exhibit a rectangular shape, with its long axis aligned along the surface dimer row direction. In total we have determined the aspect ratio of 359 islands from five different experiments. The maximum in the probability distribution diagram of the aspect ratio occurs at an aspect ratio of only $1: 1.4$, which is much smaller than the aspect ratio one should expect from the ratio of the step-free energies (i.e., $\sim 1: 2$ at $500 \mathrm{~K}$ ). We ascribe this difference to the influence of the strain energy due to the presence of an anisotropic surface stress on the equilibrium vacancy island shape.

In order to address the effect of surface stress anisotropy on the equilibrium (vacancy) island shape we first show that in the absence of surface stress the aspect ratio of an (vacancy) island is simply determined by the ratio of the step-free energies. For simplicity we consider here a

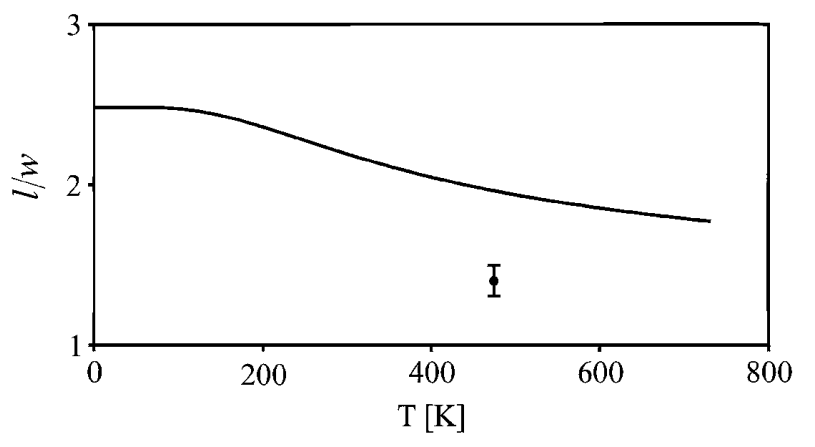

FIG. 2. Aspect ratio $(l / w)$ of a vacancy island on $\mathrm{Ge}(001)$ versus $T$ in the absence of surface stress anisotropy. The experimental obtained aspect ratio with error bar $(1.4 \pm 0.1)$ is also shown. 


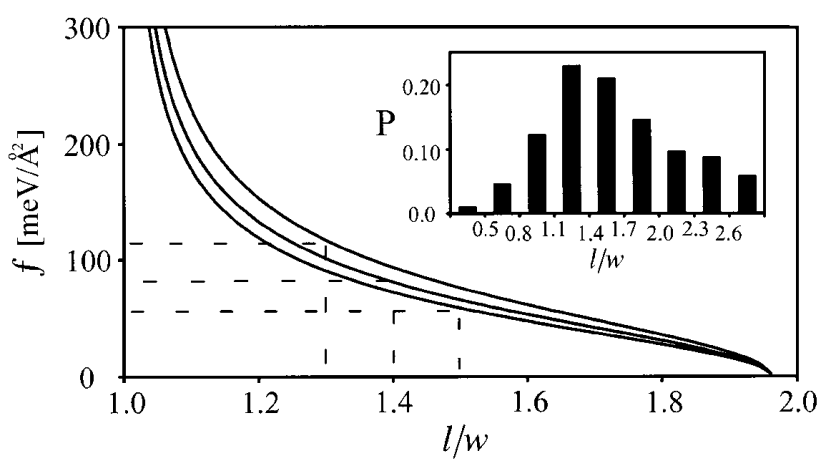

FIG. 3. Anisotropy of the surface stress, $f$, versus the aspect ratio of a vacancy island (lower curve, vacancy island size of $24 D / a_{0}$; middle curve, vacancy island size of $48 D / a_{0}$; upper curve, vacancy island size of $\left.96 D / a_{0}\right)$. The inset shows the distribution of the aspect ratio of the vacancy islands.

two-dimensional vacancy island that has a rectangular shape of length $l$ and width $w$ on a (001) surface of a material exhibiting the diamond structure. The total excess free energy, $F_{\text {tot }}$, of a vacancy island with length $l$ and width $w$ is fully governed by the total edge-free energy, i.e.,

$$
F_{\text {tot }}=2 l F_{A}+2 w F_{B},
$$

where $F_{A, B}$ are the step-free energies per unit length for a step edge along the substrate dimer row direction $(A$ step) or perpendicular to the substrate dimer row direction ( $B$ step). The step-free energies depend strongly on the temperature according to

$$
F_{A, B}=\gamma_{A, B}-k T \ln \left(\frac{1+e^{-\varepsilon_{A, B} / k T}}{1-e^{-\varepsilon_{A, B} / k T}}\right),
$$

where $\gamma_{A}=17 \mathrm{meV} / a$ ( $a$ is the surface lattice constant, i.e., $4 \AA$ ) and $\gamma_{B}=43 \mathrm{meV} / a$ are the step edge formation energies, and $\varepsilon_{A}=43 \mathrm{meV} / a$ and $\varepsilon_{B}=17 \mathrm{meV} / a$ [27] are the kink creation energies (here we have used a step edge edge freeze in temperature of $500 \mathrm{~K}$ rather than the $575 \mathrm{~K}$ used in Ref. [27]). Minimization of the excess free energy per unit area while keeping the island size $(l w)$ constant results in an equilibrium shape of the vacancy island which is determined by the ratio of the step-free energies, i.e., $\frac{l}{w}=\frac{F_{B}}{F_{A}}$.

In Fig. 2 a plot of the equilibrium vacancy island size versus temperature is depicted. At zero temperature the aspect ratio is $1: 2.5$, whereas with increasing temperature the vacancy island shape becomes slightly more compact (at $800 \mathrm{~K}$ the aspect ratio is about $1: 1.8)$. After removal of a few percent of the surface dimers on $\mathrm{Ge}(001)$ by argon-ion

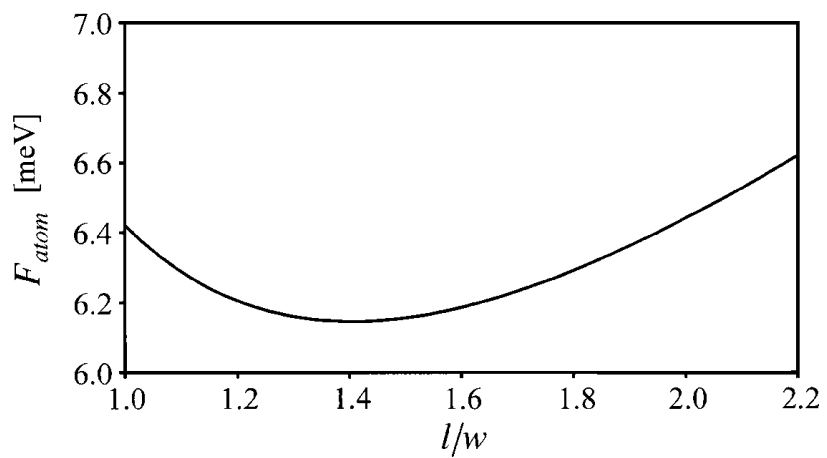

FIG. 4. Excess surface-free energy per atom versus the aspect ratio of a vacancy island for $f=80 \mathrm{meV} / \AA^{2}$.

bombardment and subsequent annealing at a temperature of $500 \mathrm{~K}$ for $1 \mathrm{~min}$ we find that the aspect ratio of the vacancy islands is only 1:1.4. After having discussed the aspect ratio of the equilibrium shape of a vacancy island in the stress-free case, we now make contact with a model recently put forward for a biaxially strained (001) surface by Li et al. [26]. Li et al. have pointed out that the equilibrium shape of a (vacancy) island under stress is determined by both island step-free energies and strain energies. If one assumes a force density $|\mathbf{f}|\left(\mathbf{f}=\sigma_{l}-\sigma_{w}\right.$; along the $A$-type edges the force density is positive, whereas the force density is negative along the $B$-type edges) along the periphery of the island edges the excess free energy per unit area can be written as

$$
\begin{aligned}
F= & \frac{2 F_{A}}{w}+\frac{2 F_{B}}{l}+\frac{2(l+w)}{l w} \\
& \times E_{S}\left[G\left(\sqrt{\frac{l}{w}}\right)-2(1-\nu) \ln \frac{\sqrt{l w}}{a_{0}}\right] \\
F= & \frac{2 F_{A}}{D^{2}} l+\frac{2 F_{B}}{l}+2\left(\frac{l}{D^{2}}+\frac{1}{l}\right) \\
& \times E_{S}\left[G\left(\frac{l}{D}\right)-2(1-\nu) \ln \frac{D}{a_{0}}\right]
\end{aligned}
$$

where $E_{s}=\frac{1+\nu}{2 \pi \mu} f^{2}$ is the unit strain energy, representing the interaction energy of two parallel force monopoles at unit separation, $\mu\left(=1.03 \times 10^{11} \mathrm{~N} / \mathrm{m}^{2}\right)$ and $\nu(=0.28)$ are the Young modulus and Poisson ratio of the substrate, respectively, $a_{0}$ is a cutoff length in the range of the surface lattice constant [26], and $D=\sqrt{l w}$ is the square root of the island size. $G(l / d)$ is a dimensionless geometric factor which depends on the island aspect ratio $c^{2}\left(c=\frac{l}{D}=\right.$ $\sqrt{l / w}$ ) as follows:

$$
\begin{aligned}
G(c)= & \frac{1}{2\left(c+\frac{1}{c}\right)}\left[2(1-\nu)\left(c \ln \frac{\sqrt{c^{2}+\frac{1}{c^{2}}}+c}{\sqrt{c^{2}+\frac{1}{c^{2}}}-c}+\frac{1}{c} \ln \frac{\sqrt{c^{2}+\frac{1}{c^{2}}}+\frac{1}{c}}{\sqrt{c^{2}+\frac{1}{c^{2}}}-\frac{1}{c}}-2 c \ln \frac{c}{e}-2 \frac{1}{c} \ln \frac{1}{c e}\right)\right] \\
& +\frac{2}{\left(c+\frac{1}{c}\right)}\left[(1-4 \nu)\left(c+\frac{1}{c}\right)-2(1-3 \nu) \sqrt{\left(c^{2}+\frac{1}{c^{2}}\right)}\right] .
\end{aligned}
$$


One eventually finds

$$
F=\frac{1}{D}\left(2 F_{A} c+\frac{2 F_{B}}{c}\right)+\frac{1}{D}\left(c+\frac{1}{c}\right) E_{s}\left(G(c)-2(1-\nu) \ln \frac{D}{a_{0}}\right) .
$$

A minimum in the excess free energy per unit area for a fixed (vacancy) island size is found by setting $\left(\partial F_{\text {atom }} / \partial c\right)_{D}=0$ [and requiring that $\left(\partial^{2} F_{\text {atom }} / \partial c^{2}\right)_{D}>0$ ]. In this minimum, one finds

$$
f^{2}=\frac{2 \pi \mu}{(1+\nu)} \frac{\left(\frac{F_{B}}{c^{2}}-F_{A}\right)}{\left(1-\frac{1}{c^{2}}\right)\left(G(c)-2(1-\nu) \ln \frac{D}{a_{0}}\right)+\left(c+\frac{1}{c}\right) \frac{d G}{d c}} .
$$

In Fig. 3 a plot of the anisotropy of surface stress tensor (i.e., $f$ ) versus the square root of the vacancy island aspect ratio for island sizes of 24,48 , and $96 D / a_{0}$ units is given. For vanishing $f$ the original result, i.e., the aspect ratio of the vacancy island is directly given by the ratio of the step-free energies, is of course recovered. Interestingly, for fixed surface stress anisotropy the aspect ratio of a vacancy island increases only slightly with increasing vacancy island size. Using Fig. 3 we can find immediately that a value for $f$ of $80 \mathrm{meV} / \AA^{2}$ is necessary to explain the experimentally observed aspect ratio of 1:1.4. It should be noted that here the actual value of $f$ we extracted from Fig. 3 is very insensitive to the exact value of the microscopic cutoff length $a_{0}$ (in the range of the surface lattice constant, i.e., about $4 \AA$ ). Finally, in Fig. 4 a plot of the excess surface free energy per atom (area $4 \AA \times 4 \AA$ ) versus the vacancy island aspect ratio is shown for $f=80 \mathrm{meV} / \AA^{2}$. The minimum of the excess surface free energy per atom versus the vacancy island ratio is quite shallow, implying that a broad distribution in the vacancy island ratio is expected. The latter result is in agreement with the experimental observations.

In conclusion, by analyzing the equilibrium shape of vacancy islands on $\mathrm{Ge}(001)$ we have extracted the anisotropy of the surface stress tensor. The aspect ratio of an island or vacancy island depends on the relative strengths of the step-free energies and the strain energy. Since the $\mathrm{Ge}(001)$ step-free energies are known, the strain energy and thus the surface stress anisotropy can be derived from the measured equilibrium shape of the vacancy islands. We have found a surface stress anisotropy of the $\mathrm{Ge}(001)$ surface of $80 \pm 30 \mathrm{meV} / \AA^{2}$ which is comparable to the surface stress anisotropy of the closely related $\mathrm{Si}(001)$ surface. This is an intriguing and important result because the local parameters of $\mathrm{Ge}$ and $\mathrm{Si}(001)$ surface, such as buckling angle and exchange integral, describing the dimer-dimer interaction are quite different for both surfaces.

*Corresponding author.

Email address: h.j.w.zandvliet@tn.utwente.nl
[1] J. Dąbrowski and H.-J. Müssig, Silicon Surfaces and Formation of Interfaces (World Scientific, River Edge, NJ, 2000).

[2] H. J. W. Zandvliet, Rev. Mod. Phys. 72, 593 (2000).

[3] H. J. W. Zandvliet et al., Phys. Rev. B 57, R6803 (1998).

[4] P. Bedrossian and T. Klitsner, Phys. Rev. Lett. 68, 646 (1992).

[5] P. Bedrossian and T. Klitsner, Phys. Rev. B 44, 13783 (1991).

[6] H. J. W. Zandvliet et al., Phys. Rev. B 46, 7581 (1992).

[7] H. Feil et al., Phys. Rev. Lett. 69, 3076 (1992).

[8] P. Bedrossian, Surf. Sci. 301, 223 (1994).

[9] K. Wurm et al., Phys. Rev. B 50, 1567 (1997).

[10] Y. Wei, L. Li, and I. S. T. Tsong, Appl. Phys. Lett. 66, 1818 (1995).

[11] D. Rioux et al., Phys. Rev. B 49, 11071 (1994).

[12] D. Rioux et al., Phys. Rev. B 50, 4430 (1994).

[13] D. Rioux et al., Phys. Rev. B 51, 10981 (1995).

[14] H. Niehus et al., J. Microsc. 152, 735 (1988).

[15] H. J. W. Zandvliet et al., Phys. Rev. Lett. 75, 3890 (1995).

[16] V. A. Ukraintstev and J. T. Yates, Jr., Surf. Sci. 346, 31 (1996).

[17] C.S. Chang et al., Surf. Sci 367, L8 (1996).

[18] J.-Y. Koo et al., Phys. Rev. B 52, 17269 (1995).

[19] H. J. W. Zandvliet, Surf. Sci. 377, 1 (1997).

[20] M.-H. Tsai et al., Phys. Rev. B 56, 7435 (1997).

[21] E. Kim et al., Phys. Rev. B 60, 8680 (1999).

[22] P.C. Weakliem, Z. Zhang, and H. Metiu, Surf. Sci. 336, 303 (1995).

[23] F. Men et al., Phys. Rev. B 52, R8650 (1995).

[24] T. Kawabe and A. Natori, Surf. Sci. 462, 181 (2000).

[25] H. J.W. Zandvliet and E. de Groot, Surf. Sci. 371, 79 (1997).

[26] A. Li, F. Liu, and M. G. Lagally, Phys. Rev. Lett. 85, 1922 (2000).

[27] H. J. W. Zandvliet, Phys. Rev. B 61, 9972 (2000).

[28] O. L. Alerhand et al., Phys. Rev. Lett. 61, 1973 (1988).

[29] F.-K. Men et al., Phys. Rev. Lett. 61, 1469 (1988).

[30] B. S. Swartzentruber et al., Phys. Rev. B 47, 13432 (1993).

[31] H. J. W. Zandvliet and H. B. Elswijk, Phys. Rev. B 48, 14269 (1993).

[32] J. Dąbrowksi, E. Pehlke, and M. Scheffler, Phys. Rev. B 49, 4790 (1994).

[33] A. García and J.E. Northrup, Phys. Rev. B 48, 17350 (1993). 